\title{
Oficina sin papeles: experiencia en una universidad venezolana
}

José Ramón Camacho Camargo

Universidad Nacional Experimental Sur del Lago

Venezuela

Jeanette Margarita Monsalve

Universidad Nacional Experimental Sur del Lago

Venezuela

Eldimar José Larez

Universidad Nacional Experimental Sur del Lago

Venezuela
Recibido: 30 de septiembre de 2014

Aceptado: 1 de diciembre de 2014

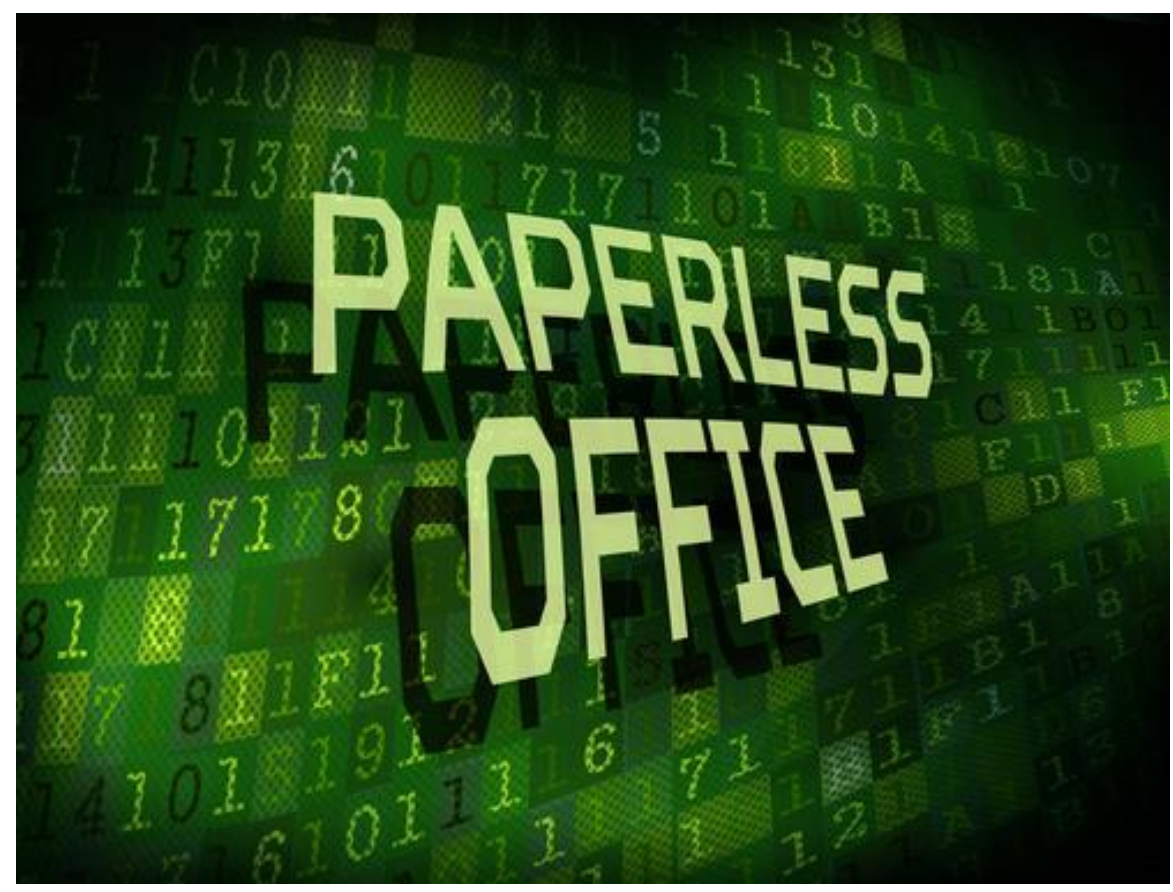

Imágenes utilizadas bajo licencia de uso por Shutterstock.com

\begin{abstract}
Excessive consumption of paper and poor management of documentation are weaknesses that may have a significant impact on productivity of organizations. For this reason, he began to develop the initiative to implement the concept of "paperless office" in the "Núcleo La Victoria de la Universidad Nacional Experimental Sur del Lago (UNESUR)" in Venezuela, part of their academic processes, particularly in the related "Grades and Diplomas Section". The research conducted was descriptive and feasible project. The proposal identified resource savings associated with implementing this practice. The results indicate that it is feasible to implement and which has a major impact on productivity; however, must be accompanied by measures for backup and information security, so its application in other areas of the university organization will be proposed, which can serve as an example for application in business organizations.
\end{abstract}

KEYWORDS: Virtual office, productivity, eco-office 



\section{RESUMEN}

El consumo excesivo de papel y la deficiente gestión de la documentación son debilidades que pueden tener una incidencia importante en la productividad de las organizaciones. Por tal razón, se empezó a desarrollar la iniciativa de implementar el concepto de "oficina sin papeles" en el Núcleo La Victoria de la Universidad Nacional Experimental Sur del Lago (UNESUR), en Venezuela, en parte de sus procesos académicos, concretamente en los relacionados con la "Unidad de Trabajo de Grado". La investigación realizada fue de tipo descriptivo y proyecto factible. La propuesta determinó el ahorro en recursos que implica la aplicación de esta práctica. Los resultados indican que es viable su implementación y que tiene un impacto importante en la productividad; sin embargo, debe acompañarse de medidas para el respaldo y seguridad de la información, por lo cual se propondrá su aplicación en otras áreas de la organización universitaria, lo que puede servir como ejemplo para su aplicación en organizaciones empresariales.

PALABRAS CLAVES: Oficina virtual, productividad, eco-oficina

\section{Introducción}

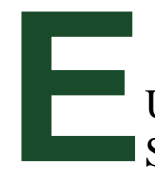

$\mathrm{n}$ el Núcleo La Victoria de la Universidad Nacional Experimental Sur del Lago se desarrolló la propuesta de implementación del proyecto oficina sin papeles. Entre los propósitos de la iniciativa está el de servir como centro piloto de la idea, en la que se pueda desarrollar una metodología de prevención en la generación de residuos y la reducción del consumo de materia prima, materiales y equipos mediante el uso de la tecnología disponible y el cambio en el comportamiento de los trabajadores.

A partir del Manual de implantación de la oficina sin papeles de la Junta de Castilla y León (2009), se planteó como objetivos identificar procesos claves, poner de manifiesto las ventajas y dificultades de la implementación en relación con el medio ambiente, las nuevas tecnologías y la reducción de papel. Para la ejecución del proyecto oficina sin papeles, se seleccionó la Unidad de Trabajo de Grado, se desarrolló la propuesta y se determinó el ahorro de papel por la aplicación del concepto de oficina sin papeles.

\section{Fundamentación teórica}

La Universidad Nacional Experimental Sur del Lago "Jesús María Semprum" tiene su sede principal en la ciudad de Santa Barbará de Zulia y extensiones en diferentes sitios del Sur del Lago de Maracaibo y valle del Mocoties en Venezuela. Uno de sus Núcleos se encuentra en el municipio Antonio Pinto Salinas del estado de Mérida. La mayoría de sus estudiantes están residenciados en las poblaciones que conforman el Valle del Mocotíes y el valle de El Chama. De igual manera, los trabajadores tienen su lugar de residencia en esas poblaciones. Por encontrarse las instalaciones universitarias lejos de zonas urbanas, es costoso para los estudiantes el traslado para realizar solo trámites administrativos.

Otro elemento especial de la ubicación del núcleo La Victoria, es que se encuentra funcionando en las instalaciones de la antigua Hacienda La Victoria. Esta es una antigua casona de estructura colonial que estuvo dedicada al procesamiento de café. Actualmente en ella funcionan dos museos, el Museo del inmigrante y el Museo del café bajo la administración de la Corporación Merideña de Turismo. Además, funciona el Núcleo universitario como parte de la Universidad Nacional Experimental Sur del Lago. El espacio asignado para el funcionamiento de oficinas es muy escaso. Por otra parte, no se pueden realizar modificaciones a la estructura, por ser parte del patrimonio cultural del estado de Mérida. Ello hace que el núcleo no tenga espacio físico para la ubicación de archivos y el desarrollo de las actividades relacionadas con la gestión de documentación.

Además, el proceso de la unidad curricular y administrativa Trabajo de grado genera una gran cantidad de documentación para lo cual se requiere el uso de archivos y espacio de 
almacenamiento. Estos se congestionan rápida y fácilmente, y se deterioran debido al peso de los documentos, lo que ocasiona en algunas ocasiones situaciones de riesgo para los usuarios que requieren información en estos archivos.

Otra de las particularidades del Núcleo universitario La Victoria se refiere a su ubicación geográfica; sus vías de acceso están expuestas a ser afectadas por la ocurrencia de eventos hidrometereológicos (derrumbes y deslaves ocasionados por las lluvias) que ocasiona retrasos y paralizaciones en sus actividades académicas y administrativas. Por esta razón, se estudió la posibilidad de gestionar algunos de los procesos de trabajo utilizando las herramientas informáticas de programas informáticos gratuitos que proporciona la compañía Google. Para esto se tomó como área piloto la Unidad de Trabajo de Grado.

Como parte de la formación profesional que recibe el estudiante de la Universidad Nacional Experimental Sur del Lago, está la formación en investigación. Uno de los requisitos de egreso lo conforma la presentación de un trabajo especial de grado. Los procesos relacionados con este requisito están bajo la responsabilidad de la Unidad de Trabajo de Grado.

En esta unidad se llevó a cabo la aplicación del concepto de oficina sin papeles. Este es un concepto nuevo, difícil de implementar en organizaciones tradicionales que apoyan sus procedimientos en una cultura organizacional que utiliza el papel como soporte de las operaciones. Es una iniciativa para aplicar las tecnologías de información y las prácticas ambientales que contribuyan con el desarrollo sustentable y con el mejoramiento de los procesos de trabajo de la institución, para optimizar con ello el uso de los recursos y así obtener mayores niveles de eficiencia, y todo esto implica un cambio en los procesos de trabajo de las organizaciones.
La oficina sin papeles como concepto aparece con la llegada de los ordenadores personales, pero su implementación se vio retrasada debido al uso de los sistemas de impresión y fotocopiado. El desarrollo de internet ha favorecido que se retome este concepto, además del impacto que puede tener sobre los temas ambientales. En un estudio de Gartner Group (citado en Doknos, n.d.) sobre gestión documental en organizaciones norteamericanas realizado en el año 2001, se estimó que:

- En promedio, las organizaciones gastan $\$ 120$ en mano de obra para encontrar un documento mal archivado.

- Estiman que un 7,5\% de los documentos se pierden.

- El 3\% se extravía por estar mal archivado.

- Los empleados asignados al área pasan $15,5 \%$ de su tiempo en lectura de información que contienen los documentos, pero hasta un $50 \%$ buscando dentro de ellos.

- El documento promedio es copiado 19 veces.

- El 30\% de los documentos en papel están fuera de fecha antes de su distribución.

- La gestión de reclamos es de 40 a $60 \%$ del tiempo de oficinistas y entre 20 a $40 \%$ de los costes laborales.

- Un $70 \%$ del trabajo de los empleados se gasta en el procesamiento de documentos.

El uso de aplicaciones informáticas para la gestión documental facilita los procesos de archivo, localización y generación de información, lo que representa una gran ventaja frente a los procedimientos tradicionales de gestión documental que se basan en el uso de papel y soportes físicos. Uno de los factores en contra para la implementación del concepto de oficina sin papeles tiene que ver con los procesos de trabajo que hacen uso, requieren o están relacionados con documentos legales o jurídicos y con documentos de tipo financiero. Sin embargo, con el uso y mejoramiento de tecnologías como la firma electrónica y la 
factura electrónica, esta resistencia al cambio puede ser aminorada.

En el caso de las instituciones universitarias, la oficina sin papeles puede ser implementada para prácticamente la totalidad de los procesos académicos, en los que por general, no se encuentran involucrados documentos relacionados con facturas y otros de carácter mercantil. Por otra parte es de resaltar que el marco legal venezolano favorece la aplicación de este tipo de iniciativas. En el preámbulo de la Constitución de la República Bolivariana de Venezuela del año 1999 se indica que el Estado debe promover la protección del equilibrio ecológico y establece como deberes y derechos ambientales de cada generación, un ambiente seguro, sano y ecológicamente equilibrado.

Igualmente, existe la Ley sobre simplificación de trámites administrativos, cuya finalidad expresada en su artículo 4 busca racionalizar y optimizar las tramitaciones de los ciudadanos y organizaciones ante la administración pública para mejorar su eficacia, pertinencia, utilidad, celeridad, reducir gastos operativos, obtener ahorros presupuestarios y mejorar las relaciones del estado con la población. Esta ley adicionalmente señala que los organismos de la administración pública deben:

- Elaborar planes de simplificación.

- Suprimir trámites innecesarios que incrementan: costos, ineficiencia y conductas deshonestas de funcionarios.

- Utilizar al máximo los elementos tecnológicos de los que se disponga actualmente.

- Incorporar controles automatizados.

- Implementar bases de datos de fácil acceso (Art. 23).

Además señala que cada organismo de la administración pública debe crear un sistema de información centralizada, automatizada, ágil, de fácil acceso, de atención al público y habilitar sistemas de transmisión electrónica de datos.

Adicionalmente existe el Decreto mediante el cual se declara el acceso y el uso de internet como política prioritaria para el desarrollo cultural, económico, social y político de la República Bolivariana de Venezuela $\left(\mathrm{N}^{\circ} 825\right.$ 10/05/2000). También el Plan nacional de telecomunicaciones, ordenación del territorio, de desarrollo regional, señala el uso de internet a todos los niveles para:

- Mejorar la calidad de vida de la población.

- Prestar servicios eficientemente de diversa índole a los ciudadanos.

Los órganos de la Administración Pública deben desarrollar sus actividades con el uso del Internet para facilitar la tramitación de los asuntos de sus respectivas competencias y el intercambio de información con particulares y la comunidad. La Ley orgánica de administración central también incluye disposiciones que favorecen el uso de la implementación de las tecnologías de comunicación e información entre las que se encuentra proyectos como la oficina sin papeles, concretamente en sus artículos 9, 72 y 74. Asimismo existen compromisos internacionales tales como: la carta mundial de la naturaleza (1982), la declaración sobre el derecho al desarrollo (1986), la conferencia de las Naciones Unidas sobre el medio ambiente y el desarrollo (1992), y la carta de la tierra (2000).

La aplicación del concepto de oficina sin papeles presenta ventajas para las organizaciones que decidan implementarla. Entre las principales se encuentran la disminución en el consumo de papel, lo que genera a su vez la simplificación de los procesos de almacenamiento y recuperación de la información. Igualmente hay una disminución notable en los costos de consumibles de impresión, como tóner, cartuchos de impresión, tintas, fotocopias, entre 
otros. Otra ventaja notable se refiere a la liberación de espacio en las oficinas con la disminución de archivos y muebles destinados al almacenamiento, con un ahorro importante en el costo de adquisición y mantenimiento de los mismos.

\section{Objetivos}

Para la ejecución de la propuesta se diseñaron los siguientes objetivos:

1. Describir el proceso de trabajo de grado aplicado en la Universidad Nacional Experimental Sur del Lago.

2. Diseñar el proceso de trabajo de grado haciendo uso de software de distribución gratuita.

3. Determinar la factibilidad de implementación de la propuesta de oficina sin papeles en la Universidad Nacional Experimental Sur del Lago.

4. Desarrollar el proceso de trabajo de grado aplicando la propuesta de oficina sin papeles.

5. Determinar el ahorro de recursos que se obtiene con la implementación de la oficina sin papeles.

\section{Metodología}

La investigación fue de tipo descriptiva, se basó en el análisis, registro y descripción de las características más trascendentales del estudio, y tomó en consideración el problema planteado referido a oficina sin papeles en el Núcleo La Victoria de la UNESUR, y como proyecto factible, el cual consiste en una "proposición sustentada en un modelo operativo viable, orientado a resolver un problema planteado o a satisfacer necesidades en una Institución o campo de interés nacional" (Universidad Nacional Experimental Simón Rodríguez, 1980, p. 79). Asimismo implica proponer alternativas de cambio con el diseño o creación de algo (Hurtado de Barrera, 1998).
Esta modalidad se presenta por la necesidad de incorporar una solución al problema de inexistencia de espacio para archivar la documentación y la movilización de trabajadores y estudiantes por la ubicación geográfica en que se encuentra el Núcleo para la entrega de documentación. Esta fase permitió determinar el consumo de recursos que genera la aplicación del proceso tradicional basado en el uso de papel como soporte de la documentación.

Posteriormente, se analizaron los programas informáticos disponibles que pudieran servir de apoyo a la implementación de la propuesta de oficina sin papeles. La Universidad Sur del Lago utiliza la plataforma de la compañía Google en el uso de los correos institucionales. Se utilizaron las herramientas que conforman esta plataforma para dar apoyo a la propuesta. Concretamente se utilizaron las siguientes aplicaciones: Gmail, Google Calendar y Google Drive.

\section{Resultados y discusión}

El Cuadro 1 señala los pasos que sigue la Unidad de Trabajo de Grado desde que el estudiante y el tutor académico llegan a un acuerdo para emprender la investigación, hasta la entrega del trabajo de grado definitivo. La primera columna señala el número de actividad, la siguiente muestra la actividad y la columna tres del cuadro indica el número de folios involucrados. Al final del cuadro se muestra el número total de folios generados por la actividad del trabajo especial de grado, este es un número aproximado por cada estudiante involucrado en el proceso.

El consumo total que genera el proceso por cada estudiante es de 992 folios. Lo que implica el número de folios que contienen dos resmas de papel tipo carta. Este número no incluye los borradores de avance que el tutor, jurado y asesores le pueden solicitar al estudiante. Por lo tanto, el número anterior sería el monto mínimo de folios involucrados en el proceso. 
Cuadro 1. Consumo de papel ocasionado por el proceso de trabajo de grado por estudiante

\begin{tabular}{lll}
\hline $\mathbf{N}^{\mathbf{0}}$ & Actividad & $\mathbf{N}^{\mathbf{0}}$ de folios \\
\hline 1 & Aceptación de tutor & 3 \\
2 & Postulación & 3 \\
3 & Entrega de postulación a jurados & 9 \\
4 & Devolución de observaciones & 12 \\
5 & Entrega de observaciones al estudiante & 5 \\
6 & Entrega de proyecto de trabajo de grado & 122 \\
7 & Entrega de proyectos a jurados y suplentes & 12 \\
8 & Entrega de correcciones de jurados & 9 \\
9 & Entrega de correcciones a estudiante & 5 \\
10 & Entrega de trabajo final a "Unidad de Trabajo de & \\
11 & Grado" & 402 \\
12 & Entrega de trabajo final a jurados & 8 \\
\hline & & 402 \\
\hline
\end{tabular}

Para la implementación de la oficina sin papeles, se utilizó la plataforma de Google, ya que la institución maneja sus cuentas institucionales utilizando este software. Se solicitó la apertura de una cuenta institucional para la Unidad de Trabajo de Grado del núcleo La Victoria. En la misma se abrieron carpetas para el control de la documentación enviada y recibida, postulaciones por semestre, etc.

Esta plataforma posee una aplicación denominada "Google Drive", este es un disco virtual, que otorga una capacidad gratuita de almacenamiento de 15 GB (aunque constantemente está aumentando). Este conjunto de aplicaciones se pueden gestionar a través de una cuenta de correo de google. Haciendo uso de esta aplicación, con la cuenta institucional de correo de la Unidad de Trabajo de Grado del núcleo, se abrieron dos carpetas denominadas Postulaciones Administración y Postulaciones Ingeniería.

Dentro de estas carpetas, se abren subcarpetas identificadas con el semestre que se esté cursando y dentro de estas carpetas, se abren subcarpetas con la documentación de cada estudiante. Este esquema de carpetas representaría las gavetas de un archivo, que contiene la documentación del proceso de trabajo de grado. Todos los documentos relacionados con el proceso de trabajo de grado se manejan en formato digital.
Las comunicaciones que genera la Unidad de Trabajo de Grado son enviadas a los profesores y estudiantes por medio del correo electrónico, el proyecto de trabajo de grado es enviado también por esta vía a los jurados, las observaciones al proyecto de trabajo de grado y a la versión definitiva también se realizan vía digital. El informe de gestión de la Unidad de Trabajo de Grado también se realiza de manera digital, todas las comunicaciones, avisos y cronogramas que elabora la Unidad de Trabajo de Grado son realizadas utilizando medios digitales.

La puesta en práctica de la digitalización del proceso generó una reducción de sesenta por ciento en el uso de papel. Sin embargo, puede generar la reducción del uso de papel hasta en un noventa por ciento, en caso de que se solicite al estudiante una sola impresión de su trabajo definitivo de grado.

Aparte de la reducción en el consumo de papel, se redujo la actividad de impresión, el consumo de cartuchos de tinta, disminución de electricidad, ahorro de tiempo, traslados de los estudiantes, tutores y jurados a la institución para realizar los trámites, entre otros. Se tiene previsto a corto plazo aplicar esta técnica en el proceso de pasantía de la institución.

La reducción de papel conllevó la disminución de otros elementos como lapiceros, sellos, sobres, entre otros que finalmente se convierten en desperdicios. La aplicación del concepto de oficina sin papeles ha permitido mejorar la eficiencia y contribuido a la eficacia en la organización.

Para su implementación, se contó con la ventaja de que el personal encargado del proceso de trabajo de grado ya tenía un conocimiento previo de las herramientas. En otros casos, se requiere el entrenamiento $y$ capacitación en el manejo de este tipo de herramientas virtuales (Fernández, Fernández y Romero, 2004). 
Sin embargo, el manejo de estas herramientas sólo requiere de conocimientos básicos de informática, por lo que no es complicado su aprendizaje y dominio. Igualmente la recepción de los estudiantes ha sido positiva con el proceso. Un elemento a favor de la implementación de este proceso es que los estudiantes manejan las herramientas utilizadas con mucho conocimiento y destreza.

El desarrollo del proceso de trabajo de grado basado en la digitalización de los documentos contó con el apoyo y aprobación de parte de los estudiantes como usuarios de dicho proceso, ya que este implica, en el caso concreto del Núcleo La Victoria, una disminución de los traslados hacia la institución por parte del estudiante, y es un beneficio para ellos gestionar la documentación desde sus diferentes sitios de residencia. De esta manera, se obtienen ahorros significativos en el costo de transporte y en el tiempo dedicado al traslado, y así pueden disponer de dicho tiempo para otras actividades.

Sin embargo, a pesar que la información que se maneja se encuentra localizada en los equipos utilizados y adicionalmente se utilizan discos virtuales que permiten compartirla $y$ resguardarla, es importante complementar esta práctica con procesos adicionales orientados al respaldo de la misma, mediante programas gratuitos de libre distribución y acceso por internet. El respaldo se haría en otro tipo de soportes, siendo la grabación en discos tipo DVD una alternativa viable para organizaciones que no manejen una gran cantidad de datos y para las organizaciones pertenecientes al sector de las pequeñas y medianas empresas.

Es de importancia para la organización enfocarse en el manejo de la información y no en el uso de la tecnología de información. La importancia de esta tecnología de información radica no en la tecnología en sí, sino en el acceso a la información, el conocimiento y las comunicaciones (Arribas Urritia, 2000). Es de resaltar que para la digitalización del proceso de trabajo de grado no se utilizó ningún programa especial de gestión de la documentación, lo que implica el ahorro en pago de licencias y la inversión en capacitación y posiblemente en adquisición de equipo. Este caso se utilizó un programa de libre acceso cuyo uso no involucra costos adicionales para la organización ni para el estudiante.

Entre los beneficios adicionales se encuentra una mejora en la búsqueda de expedientes, las respuestas de una forma más inmediata a los estudiantes, además del ahorro de espacio y la disminución del consumo de papel. La propuesta es viable. No requiere inversiones adicionales en equipos y la posible capacitación requerida por el personal implicaría una baja inversión ya que tanto este como el estudiantado posee las destrezas necesarias para el uso de las herramientas que utiliza la propuesta.

Se tiene previsto la elaboración de un plan que permita implantar el concepto de ecooficina. La propuesta de oficina sin papeles sería uno de los programas del plan, el cual debe incluir la elaboración de un Manual de buenas prácticas en la oficina que guíe a los trabajadores para el desarrollo de conductas que favorezcan el ambiente al tiempo que se favorece la eficiencia, productividad y eficacia. Igualmente se complementará con procesos orientados al respaldo de la información en soportes distintos a la nube, para lo cual se evaluará la relación costo/beneficio de dichos procesos. Por último, se pretende integrar a todos los departamentos en el uso de las tecnologías y los beneficios que traen.

\section{Conclusiones}

La puesta en práctica del concepto oficina sin papeles demuestra que su aplicación es viable en las organizaciones. Puede representar una alternativa para la reducción de costos y el mejoramiento de productividad, con una mayor integración con la organización a través del entrenamiento del personal en el manejo de la tecnología necesaria para el desarrollo del concepto. Por otra parte, la implementación de 
este concepto por parte de las organizaciones puede ser utilizada como parte de las estrategias de imagen corporativa y de responsabilidad social empresarial.

La oficina sin papeles es un concepto que no se limita solo a las organizaciones. También puede ser implementado por cada individuo para el manejo de su documentación personal, presentando los mismos beneficios indicados anteriormente con su aplicación en la organización. Su aplicación puede tener un impacto significativo en el incremento de la productividad personal, lo cual puede trasladarse al trabajo realizado en la organización, ya que al mejorar la productividad personal se incrementa al mismo tiempo la productividad organizacional.

Entre las preocupaciones a las que tiene que hacer frente la gerencia de las organizaciones está el mejoramiento continuo, el incremento de la productividad organizacional, el logro de una mejor gestión ambiental, entre otros. Esto se puede lograr a través de un uso más adecuado de los recursos, constituyendo el enfoque de oficina sin papeles, una práctica que permitiría esta mejora con los beneficios adicionales de integración del personal y simplificación de los procesos de trabajo, y mediante el aprovechamiento del conocimiento del personal en lo referente al uso de redes sociales y el concepto de computación en la nube.

\section{Referencias}

Arribas Urritia, A. (2000). ¿Centralizar o descentralizar los sistemas de información en la empresa? Revista latina de comunicación social, 3(31). Recuperado de http://www.ull.es/publicaciones/latina/aa2000kj1 /z31j1/80amaia.htm.

Adiós al papel: bienvenida la oficina sin papeles. (Diciembre de 2007). Boletín de difusión de la estrategia de educación ambiental de Castilla y León, 69, 12-15. Recuperado de http://www.jcyl.es/web/jcyl/binarios/571/966/Bo lecin\%2069\%20Final.pdf?blobheader=applicati on $\% 2$ Fpdf $\% 3$ Bcharset $\% 3$ DUTF$\underline{8 \& \text { blobnocache }=\text { true. }}$.

Constitución de la República Bolivariana de Venezuela. (3 de marzo de 2000). Gaceta Oficial de la República Bolivariana de Venezuela, 5453.

Decreto No 6.265 Con Rango, Valor y Fuerza de Ley de Simplificación de Trámites Administrativos. (31 de julio de 2009). Gaceta Oficial de la República Bolivariana de Venezuela, 5.891.

Decreto 825 Decreto $N^{\circ} 825$, mediante el cual se declara el acceso y el uso de Internet como política prioritaria para el desarrollo cultural, económico, social y político de la República Bolivariana de Venezuela. 22 de mayo 2000.

Junta de Castilla y León. (2009). Manual de implantación de la oficina sin papeles. Consejería de Medio Ambiente

El reto de la oficina sin papel. (n.d.). Recuperado el 09 de noviembre de 2014en http://www.doknos.com/en/node/71

Fernández, C., Fernández de Pelakeis, C. \& Romero, D. (2004). La oficina en mi casa: una interpretación postmoderna de la oficina virtual para la alta gerencia corporativa del siglo XXI. Revista venezolana de información, tecnología y conocimiento, 1(2), 9-21.

Hurtado de Barrera, J. (1998). Metodología de la investigación holística. Caracas: Fundacite, Servicios y Proyecciones para América Latina.

Ley de Mensajes de Datos y Firmas Electrónicas. (13 de diciembre de 2000). Gaceta Oficial de la República Bolivariana de Venezuela, 37.076.

Universidad Nacional Experimental Simón Rodríguez. (1980). Vice-rectorado Académico, Programa de Postgrado, Alcances generales sobre técnicas andragógicas de Aprendizaje. Caracas: Universidad Simón Rodríguez.

\section{Información del autor de correspondencia}

José Ramón Camacho Camargo

e-mail: camachor@unesur.edu.ve

Administrador. Master en Administración mención Gerencia general. Universidad de Los Andes. Profesor de la Universidad Nacional Experimental Sur del Lago Núcleo La Victoria. Miembro del Grupo de estudios en agronegocios (GEAN) 